\title{
La tempête dans les romans français d'Alexandre du XII siècle: un stéréotype éclaté
}

\section{Esperanza Bermejo Larrea}

\section{(2) OpenEdition}

\section{Journals}

Édition électronique

URL : http://journals.openedition.org/studifrancesi/2026

DOI : 10.4000/studifrancesi.2026

ISSN : 2427-5856

Éditeur

Rosenberg \& Sellier

\section{Édition imprimée}

Date de publication : 1 avril 2014

Pagination : $3-21$

ISSN : 0039-2944

\section{Référence électronique}

Esperanza Bermejo Larrea, "La tempête dans les romans français d'Alexandre du xı ${ }^{\mathrm{e}}$ siècle: un stéréotype éclaté », Studi Francesi [En ligne], 172 (LVIII | I) | 2014, mis en ligne le 01 avril 2014, consulté le 17 septembre 2020. URL : http://journals.openedition.org/studifrancesi/2026 ; DOI : https://doi.org/ 10.4000/studifrancesi.2026

\section{(2) $\odot \Theta \Theta \Theta$}

Studi Francesi è distribuita con Licenza Creative Commons Attribuzione - Non commerciale - Non opere derivate 4.0 Internazionale. 


\title{
La tempête dans les romans français d'Alexandre du XII siècle: un stéréotype éclaté*
}

\begin{abstract}
The versions of the Roman d'Alexandre, composed by Thomas de Kent and Alexandre de Paris include the motif of the tempest inside the India episode, after passing through the Pillars of Hercules, that is, during the time when the hero contravenes the prohibition of the gods. Both authors amplify the model taken from the Epistola Alexandri ad Aristotelem, but they deal with it in a different way. In Thomas de Kent's version the tempest is a digressio, whereas Alexander de Paris disintegrates and allocates it in successive laisses to associate it with other motifs, either epic ones like the planctus, or fictional ones, like the mirabilia of the East. The result presents, in either case, a new, more human and vulnerable image of the legendary Alexander.
\end{abstract}

\section{Introduction}

Le motif de la tempête en mer s'enracine dans la tradition de la littérature latine, dont la poésie dresse un canevas descriptif qui partant des modèles Grecs se façonne et s'accomplit grâce aux contributions d'auteurs tels que Lucrèce, Horace, Óvide, Stace et surtout Virgile, suivant ce programme «formulaire»:

1. On atteint la haute mer et on perd de vue la côte.

2. Le temps se gâte: vents et vagues, nuages et obscurité, tonnerre et éclairs.

3. Les méfaits de la tempête, en particulier la dispersion des navires.

4. L'embellie.

5. L'arrivée au port ${ }^{1}$.

En ce qui concerne la littérature médiévale française, Grisward a soigneusement établi, à partir d'une analyse comparative des premiers textes où le motif apparaît - Brut et Le roman de Rou de Wace, le lai Eliduc de Marie de France et le Tristan de Thomas d'Angleterre -, la constitution dans le troisième quart du XII siècle d'une sorte de «fonds commun» sur le vocabulaire et les motifs de la tempête, dont l'origine se trouverait probablement chez Wace et qui a été ensuite utilisé par maints écrivains².

Récemment, ce motif de la tempête maritime a suscité un intérêt renouvelé des médiévistes, comme en témoignent les travaux de James-Raoul et ConnochieBourgne, qui nuancent le programme établi par Saint-Denis et complètent l'étude de ses fonctions. James-Raoul précise la durée de la tempête - trois jours - et ajoute comme élément constitutif l'expression des sentiments des hommes face aux violences atmosphériques: cris, effrois, prières, confusion ${ }^{3}$. D'autre part, l'apparition

\footnotetext{
* Cette étude fait partie des activités développées dans le cadre du projet de recherche FFI2009-08861 du Ministerio de Ciencia e Innovación espagnol.

(1) E. DE SAINT-DENIS, Le rôle de la mer dans la poésie latine, Paris, Klincsieck, 1935, p. 218.

(2) J. GRISWARD, «Â propos du thème descriptif de la tempête» in Mélanges de langue et de littéra-
}

ture du Moyen Âge et de la Renaissance offerts à Jean Frappier, Genève, Droz, 1970, p. 388.

(3) D. James-Raoul, «L'écriture de la tempête en mer dans la littérature de fiction, de pèlerinage et de voyage> in Senefiance. Mondes marins du Moyen Âge. Études réunies par Chantal ConnochieBourgne, 52, 2006, pp. 218-219. 
soudaine de la tempête s'avère être une ressource narrative féconde, car elle introduit une rupture dans le récit pour le relancer après l'apaisement et l'entraîner dans une direction nouvelle ${ }^{4}$. L'apaisement de la tempête correspond, d'après ConnochieBourgne, à une progression intérieure qui s'inscrit dans les desseins de la Providence'.

La tradition judéo-chrétienne reprend aussi ce motif. La Bible offre de nombreux passages où la tempête exprime la colère divine et bouleverse les éléments atmosphériques, agite la mer ou fait trembler la terre comme punition d'une faute individuelle ou collective ${ }^{6}$. L'homme médiéval conçoit donc les catastrophes naturelles comme des signes divins, ce qui explique leur présence notable dans les exempla, qui proposent des modèles de conduite à imiter ou à éviter ${ }^{7}$. La tempête signifie, finalement, un danger que le chevalier doit défier, et de ce fait elle s'assimile à l'aventure en tant qu'instrument de perfectionnement individuel, comme l'a bien démontré Köhler ${ }^{8}$.

Dans les versions françaises du XII siècle de l'histoire d'Alexandre le Grand, Le Roman d'Alexandre ou Le Roman de Toute Chevalerie de Thomas de Kent et Le Roman d'Alexandre d'Alexandre de Bernay, dit de Paris, la tempête a toujours lieu sur terre, jamais en mer, comme il arrive dans les autres romans antiques', à coup sûr parce que l'expédition du héros macédonien se déploie à travers les régions de l'Europe, l'Asie et l'Inde. L'Epistola Alexandri ad Aristotelem ${ }^{10}$, l'une des sources textuelles en langue vernaculaire, contient une description de tempête sur terre qui est reprise par Thomas de Kent, comme Gaullier-Bougassas le remarque dans son

(4) Après la tempête, Polynice découvre la ville d'Argos, dont le roi, Adraste, deviendra son allié dans la guerre contre son frère Étéocle; F. MORALebrun, éd., Le roman de Thèbes, Paris, Librairie Générale Française (coll. Lettres Gothiques), 1995, vv. 678-723. L'union amoureuse de Didon et Enée a lieu dans une grotte où ils trouvent refuge contre la tempête qui les surprend pendant la partie de chasse; J. J. Salverda De Grave, éd., Eneas, roman du XII ime siècle, Paris, Honoré Champion (coll. Les Classiques Français du Moyen Âge), 1964, vv. 1507-1517. La tempête provoque la disparition de Castor et Pollux, partis avant la guerre de Troie au secours de leur sœur Hélène ravie par Paris; L. Constans, éd., Le roman de Troie de Benoît de Sainte-Maure, New-York, Johnson Reprint, réimpr. éd. Paris, Firmin Didot et Cie (Société des Anciens Textes Français), 1909, 1968, I, vv. 5061-5088.

(5) C. Connochie-Bourgne, «L'apaisement de la tempête dans la littérature médiévale: quelques exemples» in Une Étrange Constance: les motifs merveilleux dans les littératures française et francophone, éd. F. Gingras, Québec, Presses de l'Université Laval (coll. Symposium), 2006, p. 120.

(6) Genèse 7, 10-8, 12: le déluge; Exode 9, 22 26: la grêle est le septième fléau d'Égypte; Deutéronome 28, 24; Psaumes 78, 47-48; Jonas 1,4; trois épisodes de la tempête apaisée et le Christ: Mathieu 8, 23-27; Marc 4, 35-41 et Luc 8, 22-35. Joëlle Ducos remarque que la perception des éléments naturels, avant le XIII ${ }^{\mathrm{e}}$ siècle, est plus souvent mythique qu'objective et elle relève de l'interprétation symbolique et théologique du monde. Cette perspective explique que l'on mette plutôt en relief les aspects violents. J. Ducos, La météo- rologie en français au Moyen Âge (XIII'-XIV siècles), Paris, Honoré Champion, 1998, pp. 395-397.

(7) J. BERLIOZ, «Les récits exemplaires, sources imprévues de l'histoire des catastrophes naturelles au Moyen Âge» in Sources: travaux historiques. Histoire des catastrophes naturelles. Paysages. Environnement, 33, 1993, p. 8.

(8) E. KöHLER, L'aventure chevaleresque. Idéal et réalité dans le roman courtois. Études sur la forme des plus anciens poèmes d'Artbur et du Graal. Préface de Jacques Le Goff, Paris, Éditions Gallimard (coll. NRF), 1974, pp. 77-102.

(9) Dans le roman d'Eneas la tempête en mer surprend les Troyens avant leur arrivée à Carthage; J. J. Salverda De Grave, éd., Eneas... cit., vv. 183276. Dans le Roman de Troie, les tempêtes en mer encadrent le siège et la guerre de Troie et indiquent le passage d'une partie du roman à l'autre: dans les prolégomènes de la guerre, le motif apparaît dans l'ambassade d'Anténor; L. Constans, éd., Le roman de Troie cit., T. I, vv. 3563-3573; dans la quête par Castor et Pollux de leur sœur Hélène, I, vv. 5061-5088; elle empêche l'arrivée des Grecs à Troie (t. I, vv. 5930-5966). Elle accompagne les héros grecs dans leurs «retours»: naufrage d'Ajax, fils d'Ö̈lée (t. IV, vv. 27570-27670), retour de Ménélas (t. IV, vv. 28412-28418), d'Ulysse (t. IV, vv. 28610-28618), de Néoptolème (t. IV, vv. 29185-29206).

(10) G. Bounoure et B. Serret, éds., PseudoCallisthène. Le Roman d'Alexandre. La vie et les hauts faits d'Alexandre de Macédoine, Paris, Les Belles Lettres, 1992. La Lettre d'Alexandre de Macédoine à Aristote son maître sur son expédition et la description de l'Inde figure comme appendice I (pp. 123-146). 
édition du roman ${ }^{11}$. La structure formelle de ce motif diffère un peu de celle que Saint-Denis trouve dans la poésie latine, tout d'abord parce que le scénario terrestre exclut de façon naturelle les clichés 1 . On atteint la haute mer et 5 . On arrive au port; ensuite, parce que les descriptions des météores et des dégâts se juxtaposent, d'où résulte le canevas suivant:

1. L'Eurus se lève soudainement. Dommages sur les tentes. Réaction des soldats et d'Alexandre.

2. Froid extrême.

3. Flocons de neige gros et abondants. Conséquences.

4. Pluie intense.

5. Nuage noir accompagné de flambeaux de feu. Bêtes et soldats brûlés.

6. L'embellie.

Alexandre de Paris, quant à lui, présente trois versions de ce motif. Cette contribution vise à montrer comment l'utilisation de la laisse épique en tant que procédé d'écriture du roman réécrit et renouvelle le topos de la tempête.

\section{Le Roman d'Alexandre ou Le Roman de Toute Chevalerie}

La description de la tempête s'insère dans la conquête de l'Inde et l'exploration de ses merveilles. Elle fait suite à l'échec d'Alexandre devant le paradis terrestre et précède la prise d'une ville et la rencontre avec les brahmanes, qui clôt l'épisode indien. Elle occupe les laisses 359-364, ce qui suppose un important exercice d'amplificatio par rapport au modèle latin. Thomas de Kent tient à bien cerner les limites de cet excursus et, pour ce faire, il reprend partiellement les vers 5787 et 5860, «unes neires nues», qui servent respectivement d'ouverture et de clôture de la description et déchaînent en même temps l'orage, ce qui établit déjà une première différence par rapport à l'Epistola, où le motif déclencheur est l'Eurus, le vent de l'Est, et le fragment descriptif passe plus librement dans le récit. La laisse 360 est liée à la précédente par une reprise bifurquée non littérale des vers 5786-5787 dans le vers 5790. Il se produit, d'ailleurs, une contradiction dans la répétition, car dans la première strophe la tempête vient «devers septentrion» tandis que dans la suivante elle provient «devers l'occident». La laisse 360 est tripartite. La première partie amplifie le motif de la tempête esquissée dans la laisse précédente avec l'addition d'éléments atmosphériques tels que le tonnerre et les éclairs, absents dans l'Epistola et d'autres menus détails sur les dégâts des tentes et des pavillons; la deuxième partie décrit longuement les différentes réactions d'Alexandre et de ses hommes à travers vingt vers, qui tout en restant fidèles au texte latin, permettent de mieux saisir la terreur des soldats face à l'argumentation de leur chef, d'une logique superbe grâce à la transcription des discours respectifs en style direct, au lieu des discours narrativisés utilisés par l'auteur latin. Chacun interprète les phénomènes selon ses sentiments. Tandis que les soldats effrayés y voient un juste châtiment de Dieu («bon dreit», v. 5797), pour avoir prétendu découvrir ses secrets, Alexandre démythifie la tempête expliquant son origine météorologique typique de la saison hivernale où ils se trouvent. La troisième partie raconte une seconde tempête encore plus forte, suivant un procédé de gradatio, car elle associe les «quatre element» (v. 5815), autrement dit, le cosmos entier assaille

(11) C. Gaullier-Bougassas, L. Harf-Lancner, B. Foster et I. Short, éds., Le Roman d'Alexandre ou Le Roman de toute chevalerie, de Thomas de Kent, Paris, Honoré Champion (coll. Champion
Classiques. Série «Moyen Âge»), 2003, p. 451, note 2. Toutes les références renvoient à cette édition, dont le texte est reproduit dans l'annexe I. 
l'armée grecque. C'est le tour du froid et de la neige, dont les flocons sont comparés à une toison, comme dans la source latine. Un vers de conclusion et de commentaire général du narrateur sur le grand péril qu'ils courent clôt la laisse, dans la plus pure tradition épique.

La laisse 361 amplifie la précédente et vise à intensifier le climax dramatique. Elle met en œuvre simultanément deux techniques: d'une part elle privilégie la description enrichie des météores - «grisil», «feu», «flambe» (vv. 5821-5823-5828) -, d'autre part, elle abrège la réaction des soldats et narrativise leurs paroles, qui s'intègrent dans le récit comme une suite de gestes, racontée à partir d'une énumération de verbes: «Donc prient Dampnedieu qe autre orré lur present; | Escrient e plorent, a genoilz prient meint» (vv. 5825-6). Néanmoins, elle introduit une étonnante nouveauté dans le vers de conclusion: Alexandre a peur et gémit devant tout le monde. Cette réaction témoigne la défaite du macédonien devant la météorologie hostile et la perte de sa supériorité morale à l'égard de ses hommes. Les deux laisses suivantes (362-363), inventées par l'auteur médiéval, jouent des variations sur l'angoisse d'Alexandre, à partir de l'association du motif de la description de la tempête avec celui de la prière épique.

Le vers d'intonation qui inaugure la laisse 362 adopte l'inversion épique - attribut+verbe être+nom - pour créer une atmosphère dramatique, renforcée par l'accumulation polysyndétique d'épithètes ${ }^{12}$ : «Hiduse e laide e horible fu la nue» (v. 5831). Ce vers renoue avec le vers 5787, qui marquait le début de la tempête avec l'arrivée du nuage noir, et entraîne la strophe vers le renchérissement des ravages provoqués par le feu et les flammes qui tombent du ciel, décrits déjà dans le vers 5794 , de sorte qu'on évoque un locus horribilis, un paysage infernal où «Li arbre en sunt chaeit, l'erbe est abatue, I La terre environ est ardante aparue» (vv. 5835-5836). Dans ce décor hostile poussé à l'extrême, Alexandre fait sa prière qui s'accompagne d'un autre élément théâtral bien lourd de sens: le geste protecteur d'un soldat anonyme qui couvre sa tête avec un bouclier pour le secourir devant les éléments adverses. Confronté à la tempête, le personnage d'Alexandre perd sa grandeur épique et devient un homme commun qui a besoin de ses soldats. La prière complète sa transformation et découvre un Alexandre humilié qui reconnaît sa folie et implore modestement le secours divin.

La laisse 363 développe encore les derniers vers de la précédente, et s'attache en même temps par un enchaînement différé13 au vers 5830 de la strophe 361. Les répétitions, partielles, assurent l'unité thématique d'un ensemble qui combine des motifs lyriques différents. Cette strophe, bipartite aussi, présente deux protagonistes: Alexandre, qui répète sa plainte et le geste d'élévation de son écu en signe de désespoir et d'impuissance, et Tholomé, un des douze pairs dont les paroles, dirigées au roi, occupent quasiment toute la laisse (neuf vers sur douze). L'allocution allie la douceur de la consolation à la rigueur des reproches adressés au chef qui a perdu son audace et se montre abattu et découragé par la violence atmosphérique: «Sire», dit Tholomeu, «n'aiez en quer pesance. | Ces perils, ces tormenz pernez toz en suffrance» (vv. 58465847). Tholomé réclame la résistance physique et morale d'Alexandre et prend sur soi la responsabilité de champion qui fait partie des attributions du Macédonien.

(12) L'exemple le plus connu d'inversion épique est, sans aucun doute, le vers de la Chanson de Roland «Halt sunt li pui e li val tenebrus»; M. DE Riquer, éd., Chanson de Roland, Cantar de Roldán y el Roncesvalles navarro, Barcelona, El Festín de Esopo, 1983, v. 814. Les adjectifs «hiduse», «laide» e «horible» caractérisent la tempête. Voir les textes cités dans la note 9 .

(13) Je reprends la terminologie de D. BouteT, La chanson de geste. Forme et signification d'une écriture du Moyen Âge, Paris, Presses Universitaires de France, 1993, p. 83, qui précise la définition d'enchaînement de Rychner. 
Néanmoins, ses arguments proclament la vanité des vertus chevaleresques devant cette catastrophe naturelle, qui met à nu la condition mortelle du grand conquérant et lui fait essuyer l'humiliation de se savoir vaincu par la force d'une nature hostile qui obéit aux desseins de Dieu. Vanitas vanitatum... Le discours doctrinal remplace la harangue guerrière pour exhorter à la prière comme seul geste susceptible d'être écouté et accepté par Dieu.

Le récit de l'apaisement de la tempête raconté dans la laisse 364 reproduit fidèlement le texte de l'Epistola. Après trois jours de violence atmosphérique, l'embellie permet d'enterrer les morts et de poursuivre l'expédition à travers les déserts, accompagnés des guides. La pause narrative finit, mais Thomas a renouvelé ce motif pour décrire un Alexandre battu qui accepte son impuissance et s'en remet à Dieu.

\section{3. "Le Roman d'Alexandre" d'Alexandre de Paris}

Trois termes désignent et précisent le motif de la tempête dans ce roman: «tempier», «estorbellons» et «torment» ${ }^{14}$, qui comme dans celui de Thomas de Kent s'intègrent dans la branche III sur le voyage en Inde. La description du «tourbillon» (laisses 181-187), qui pourrait convenir selon Dubost à un cyclone ${ }^{15}$, est la plus fidèle au texte de l'Epistola, surtout en ce qui concerne le choix des éléments météorologiques, dont l'ordre est parfois modifié: vent furieux, tisons de feu, neige, obscurité, averse diluvienne. Même si Alexandre de Paris emprunte à Thomas de Kent certains détails (l'ordre du héros macédonien à ses hommes de dégager la neige tombée sur le campement, qui ne disparaît complètement qu'après la pluie qui suit), son traitement et le degré d'insertion dans le récit sont fort différents. La description n'est pas réalisée d'un seul trait, elle est scindée en deux volets séparés par trois laisses $(184,185$, 186). Le premier volet décrit les météores et s'étend sur les huit derniers vers de la laisse 181 (sur un total de 23 vers), la laisse 182 (21 vers) et les trois premiers vers de la laisse 183, d'ailleurs très brève (6 vers). Le second volet décrit l'embellie dans les neuf premiers vers de la laisse 187. Ce saut dans l'espace strophique, mais aussi littéraire et temporel, est assuré par la répétition du premier hémistiche du vers d'intonation des laisses 182 et 187: «Qant li vens fu cheüs et li estorbellons» (v. 3161); «Qant li vens fu cheüs et la noif fu fondue» (v. 3254). Les laisses 184-186 narrent la rencontre d'Alexandre avec les deux vieux Indiens à longues barbes, qui l'identifient sans le connaittre et lui promettent de le guider jusqu'aux arbres du Soleil et de la Lune qui révèleront sa mort. Ils vont prendre le relais des quatre vieux qui ont montré le chemin des fontaines magiques et qui sont interrogés par Alexandre sur les causes de la météorologie extrême qui s'est abattue sur eux dans la laisse 183. Dans la laisse 186 le roi découvre la grotte enchantée par Arthur et Liber, où périssent quatre hommes fort armés. La tempête n'interrompt pas le progrès des aventures d'Alexandre; bien au contraire, toutes les deux se combinent, de sorte que le tourbillon devient une nouvelle épreuve qu'Alexandre et ses soldats doivent surmonter.

Dans ce continuum narratif, l'espace et le temps des événements sont brouillés, malgré les références à des lieux concrets. Avant le déferlement du tourbillon, Alexandre revient au point d'où il était parti, le bord d'un étang défendu par des pics

(14) L. Harf-Lancner et E. C. Armstrong, éds., Le Roman d'Alexandre d'Alexandre de Paris, traduction, présentation et notes, Paris, Librairie Générale Française (coll. Lettres Gothiques), 1994, I. 160, v. 2798; I. 181, v. 3150; I. 201, v. 3572. Les laisses citées renvoient à la branche III et sont reproduites dans l'annexe II.

(15) F. Dubost, Aspects fantastiques de la littérature narrative médiévale (XII ${ }^{\text {eme }}$-XIII ${ }^{\text {eme }}$ siècles). L'Autre, l'Ailleurs, l'Autrefois, Paris, Honoré Champion, 1991, p. 270. 
aigus et vite asséché par la soif des chevaux et des bêtes (vv. 3131-3138). C'est dans ce cadre qu'il aperçoit quelques géants poilus, dont le corps nu est fendu jusqu'au nombril, qui lancent des pierres aux soldats. Les Grecs ne peuvent pas repousser l'attaque à cause de la tempête qui les surprend et qui remplit l'étang d'eau (v. 3181); cependant, un peu plus tard la rencontre avec les vieux Indiens a lieu dans un désert vague et indéterminé (v. 3188). Ensuite, après l'ensevelissement des morts, l'armée d'Alexandre quitte un lieu élevé, «D'un grant tertre ou il ierent ont un val avalé» (v. 3218); par contre, le décor de l'embellie revient à «l'estanc acourue I Deus piés plus haut la rive de cele qu'iert beüe» (vv. 3258-3259), d'où l'armée se déplace pour monter sur un tertre de rochers pointus (v. 3264). Les Grecs avancent et rebroussent chemin, descendent pour ensuite remonter, tournent en rond comme au début de l'épisode. L'espace semble devenir circulaire pour confondre et égarer les Grecs. L'imprécision des repères chronologiques ne permet pas d'y voir clair, car les quatre jours de durée du tourbillon et les trois jours de réclusion des soldats dans la grotte d'Arthur et de Liber répondent à des clichés temporels ou symboliques.

La dissociation du topos de la tempête réunit dans son intérieur d'autres manifestations du merveilleux oriental, telles que l'humanité monstrueuse et la grotte ensorcelée $^{16}$, qui modulent le comportement du roi. Ni l'altérité étrange ni le merveilleux géomorphologique ne l'effraient, cependant la météorologie adverse, qu'il interprète comme une manifestation de la colère des dieux, l'angoisse et provoque sa fuite.

Le «tempier» fait partie de l'épisode du val périlleux, où Alexandre affronte la mort en solitaire comme sacrifice suprême pour délivrer son armée de cette vallée sans retour ${ }^{17}$, et où la succession de phénomènes naturels crée une atmosphère fantastique qui annonce la libération du diable ${ }^{18}$. La description de la tempête se distribue entre les laisses 156-160, suivant une forme disjointe comme dans le cas antérieur: entre les météores et l'embellie l'auteur glisse des planctus, les motifs lyriques connus de la chanson de geste. La répétition du premier hémistiche du vers d'intonation «Qant li rois fu remés» (1. 156, v. 2705 et 1. 158, v. 2740), et sa variante «Qant li rois vit la terre» (1. 157, v. 2719) établit une reprise horizontale parallèle entre les trois strophes qui arrête le progrès narratif et privilégie l'expression des sentiments d'Alexandre, tout en soulignant sa solitude. Les trois laisses consécutives se renferment sur elles-mêmes, comme la vallée qui emprisonne le roi. Dehors, les soldats grecs poursuivent leur chemin de retour après avoir franchi les colonnes d'Hercule. Une forte tension dramatique se crée ainsi entre l'espace intérieur et l'extérieur, entre le chef grec et ses soldats que les plaintes d'Alexandre visent à combler. Les laisses 156 et 160, ouverture et clôture respectives de la tempête, sont

(16) Pour J. Le GofF «Le merveilleux dans l'occident médiéval» in L'imaginaire médiéval: essais, Paris, Éditions Gallimard (coll. Bibliothèque des histoires), 1985, p. 36, l'Orient constitue le grand réservoir du merveilleux, parce que c'est le vrai étranger.

(17) E. Baumgartner, «L'Orient d'Alexandre» in Bien dire et bien aprandre, 6, 1988, pp. 12-13, affirme que le caractère circulaire de l'Orient dans ce roman explique l'impuissance du conquérant pour lui arracher le secret de l'immortalité, et justifie sa mort comme sacrifice suprême pour pouvoir délivrer ses hommes. C. Gaullier-Bougassas, «Le redoublement de l'enfermement d'Alexandre au val périlleux, dans le manuscrit de Venise du $\mathrm{Ro}$ - man d'Alexandre» in PRIS-MA, Le Regard II, XI, 1995 , pp. 36-37, à propos de l'épisode du pacte d'Alexandre avec le diable dans cette vallée, précise que l'auteur ne confirme pas la dimension messianique acquise par le roi dans la première partie de cet épisode, car il est incapable de se sacrifier pour toute l'humanité. Alexandre se soumet à la volonté du diable «pour reconnaître son impuissance devant une force qui le dépasse, pour mettre au jour son égoïsme et sa faiblesse qui le conduisent au mépris aussi bien du bonheur de l'humanité tout entière que du salut de son âme».

(18) F. Dubost, Aspects fantastiques... cit., pp. 272-275. 
composées à partir de ce jeu. Tandis que la météorologie pétrifie le roi, l'armée n'éprouve aucune souffrance: «A ciaus qui s'en tornerent n'avint il nul contraire» (1. 156, v. 2710). La vallée close du roi s'oppose à la mer infinie dans la longue laisse 160 (43 vers) de l'embellie: «Alixandre est el val, ne se set conseillier» (v. 2783), vers repris dans le v. 2793 «Et li rois fu el val, n'i ot que corecier», cependant «Li Grieu, qui sor la mer s'estoient fait logier (v. 2787).

La désagrégation du motif de la tempête, ainsi que les procédés de répétition de la chanson de geste, rythment l'épisode et créent une atmosphère lourde et oppressante d'une forte intensité dramatique. La laisse 157 se relie à la précédente par la reprise bifurquée du vers 2708: «Li mons prist a crauller, li vaus desous a braire» dans le vers 2720: «Les montaignes crauller et les roches movoir». Ce vers se répète aussi dans le développement de la laisse 158: «Li mons prist a crauller et li vaus a fremir» (v. 2744), et revient en écho au deuxième vers de la laisse 160: «Il ot la terre bruire, si craullent li rochier» (v. 2784). La secousse des monts et les rugissements des vallées échappent à la phénoménologie habituelle des tempêtes et évoquent plutôt un cataclysme apocalyptique ${ }^{19}$ qui menace la stabilité du globe terrestre et fait «les pierres chaoir» (1. 157, v. 2721). Néanmoins, c'est le motif le plus répété et par conséquent, celui qui marque le ton du passage. Il est suivi par la puanteur, l'un des traits qui définissent l'espace infernal dans la littérature des visions de l'au-delà ${ }^{20}:\langle E t$ gete une puor dont li rois sent la flaire» (1. 156, v. 2709), ou «Et gete unes teniebres o flairor de püir» (1. 158, v. 2745), et par l'obscurité: (1. 158, v. 2741, v. 2742 et v. 2745). La description de la tempête se complète avec d'autres motifs qui font partie de la rhétorique habituelle du topos: «touner», «foudre», «esclaire» (1. 156, v. 2707), «l'air espessier» (1. 160, v. 2796) et «les nues torbler» (1. 160, v. 2797), mais la pluie et la neige manquent. Il s'agit, donc, d'une tempête sèche.

Les principes de symétrie et de gradation régissent la construction de l'épisode de la tempête dans le val périlleux ${ }^{21}$. Les laisses 156 et 158 sont consacrées à la description des météores et suivies par les deux plaintes d'Alexandre des laisses 157 et 159. D'autre part, l'horreur de l'orage redouble avec l'apparition dans la laisse 158 des «merveilles de la Création», nom donné au bestiaire fabuleux ${ }^{22}$. Dragons, serpents volants et diables attaquent Alexandre et prétendent l'attraper avec leurs crochets de fer. Le désert indien devient l'enfer d'Alexandre. Le val périlleux ouvre la descente aux enfers qui précède la découverte d'une autre merveille, féminine cette fois mais aussi maléfique, car elle se rattache à la mort: la rencontre des «filles de l'eau» et surtout le chemin vers les fontaines de la résurrection et de l'immortalité, celle-ci hantée par le macédonien.

La tempête-cataclysme cosmique signifie un danger mortel pour le roi, qui perd son courage et même sa verticalité:

(19) Apocalypse 6, 12-14: ouverture du sixième sceau: tremblement de terre, soleil noir, lune rouge comme le sang; la montagne et l'île remuent de sa place; Apocalypse 8, 5: ouverture du septième sceau: tonnerre, éclairs, tremblement de terre; Apocalypse 8, 7: la première trompette: grêle et feu mêlé au sang; Apocalypse 11, 19: ouverture du Temple de Dieu: éclairs, tonnerres, tremblement de terre, grêle.

(20) Sur les sources orientales et la relation du Roman d'Alexandre avec les visions de l'au-delà, voir C. Gaullier-Bougassas, Les Romans d'Alexandre. Aux frontières de l'épique et du romanesque, Paris, Honoré Champion, 1998, pp. 152-154.
(21) L'utilisation des procédés rhétoriques de symétrie et de gradation comme principes de construction de l'œuvre rapprochent le roman d'Alexandre de Paris de La Chanson de Roland.

(22) Alexandre de Paris offre une relation plus longue du cortège d'animaux fabuleux, «merveilles du désert», qui s'approchent du marais la nuit pour boire de l'eau dans les laisses 63-85 de cette même branche III. Thomas de Kent offre deux versions de ce motif: la première, plus longue, occupe les laisses 285-300, où il découpe la laisse pour la faire coïncider avec la description de chaque animal; la seconde, plus brève, où les bêtes se mêlent aux hommes étranges dans les laisses 348-359. 
Desus un mabre bis, dont estoit plaine l'aire,

Sist li rois qui tous dis fu de biens essamplaire.

La paor quil destraint li change le viaire,

Car qui de mort se crient bien est drois qu'il i paire,

Tous en est enpalis, car il ne set que faire. (1. 156, vv. 2714-2718)

Dans ces circonstances, Alexandre prononce son planctus en éprouvant la séparation d'avec ses compagnons, notamment celle d'Emenidus, son gonfanonier et l'un des douze pairs, qu'il croit définitive. Alexandre de Paris précise le canevas de ce motif lyrique emprunté au répertoire de la chanson de geste et il en retient surtout les clichés 2. Apostrophe, 4. Éloge et 6. Expression de la douleur intérieure ${ }^{23}$, qu'il développe pour délaisser les quatre derniers dont la relation plus directe avec la mort conviendrait mal à l'épisode. Le roi des Grecs étale un portrait idéal d'Emenidus qui réunit toutes les qualités morales du chevalier épique et romanesque: il est puissant, sage, preux, courtois, humble, doux, sans orgueil, endurant et soutien du Macédonien. Entre toutes ces vertus se détachent la sagesse et l'humilité comme les traits distinctifs du personnage, dévoilés dans l'épisode de la branche II «La razzia de Gadres», où le comportement d'Emenidus est calqué sur celui d'Olivier dans la bataille de Roncevaux. À la fin du planctus, Alexandre partage avec ses soldats les mêmes sentiments de solitude. Dans ce désespoir, les caprices du destin sont responsables du mauvais sort des Grecs: «Hier fustes vos en joie, hui est de blanc en noir» (v. 2737). Sur cette même imprécation à la Fortune, nommée cette fois, s'ouvre la laisse 159 qui amplifie le planctus exprimé dans la laisse 157. Alexandre se proclame doublement impuissant parce qu'il est privé de son bras droit, Emenidus, et parce que les dieux l'ont confiné dans cette vallée qui lui a déclaré la guerre. Cette situation extrême le force à faire son testament spirituel et à désigner Emenidus l'héritier de son ambition pour conquérir le monde: «S'il el bien se maintient que veoir i soloie, I Tous iert sires du mont et mes cuers li otroie» (vv. 2776-2777). À la fin du planctus le roi récupère son arrogance et exprime son désir de combattre contre les dieux pour se venger de leur trahison.

Un dernier assaut de la tempête modifie le comportement d'Alexandre qui finit par demander humblement la protection des dieux, une prière qu'il accompagne du geste de couvrir sa tête d'un pan de sa pelisse. Le caractère du roi n'a pas d'équilibre et change selon les fluctuations de la tempête.

L'apaisement de la tempête dans la laisse 160 prépare la rencontre d'Alexandre avec un démon prisonnier dans ce val et délivré par le Macédonien dans un geste ambigu qui signifie la libération du roi, mais aussi la condamnation du monde au pouvoir diabolique.

La troisième tempête apparaît dans la laisse 201 et compte 53 vers. Sa longueur découvre sa fonction narrative. L'armée d'Alexandre quitte la forêt des filles-fleur,

(23) P. ZumthoR, «Étude typologique des planctus contenus dans La Chanson de Roland» in La technique littéraire des chansons de geste: actes du Colloque de Liège (septembre 1957), Paris, Belles Lettres, 1959, pp. 221-223, distingue dix clichés fixes dans la composition du motif lyrique du planctus: 1 . Lien narratif et annonce de la plainte (regreter, veï, reguarder, gesir); 2. Apostrophe; 3. Prière pour l'âme du défunt; 4 . Éloge (direct ou indirect, notamment en évoquant la misère du pays ou du lignage du défunt après sa mort); 5 . Signes extérieurs de la douleur (pasmer, plorer); 6. Expression de la douleur intérieure (duel, las, pitet); 7. Allusion à la patrie lointaine ou au lignage; 8. Topos «ubi est?»: ses proches demanderont de ses nouvelles; 9. Évocation de la situation présente (mort); 10. Mare fu(stes); voir aussi P. ZuMTHOR, «Les Planctus épiques» in Romania, 84, 1963, pp. 62-69. J.-P. Martin, Les motifs dans la chanson de geste. Définition et utilisation, Lille, Université de Lille III, Centre d'Études Médiévales et Dialectales, 1992, p. 300, précise «qu'il faudrait ajouter à cette liste la promesse de vengeance inscrite dans le cadre du planctus». 
encore une féminité négative qui empêche la pleine réalisation amoureuse du roi et de ses hommes, qui avancent guidés par les deux vieillards dans leur quête des arbres du Soleil et de la Lune. En chemin ils rencontrent une autre vallée, nouvel avatar du locus horribilis. Il s'agit d'un val fermé par quatre montagnes où règne un froid glacial; la nature y fait naître tous les ans cinq des serpents qui crachent du feu; les vents s'y affrontent et le soleil n'y brille jamais; la terre tremble et se fendille sous leurs pieds; les merveilles du désert, des couleuvres et des serpents, y abondent. Cette vallée réunit tous les éléments caractéristiques du val périlleux: clôture, obscurité, grouillement de reptiles, conditions atmosphériques extrêmes, tremblements de terre... mais elle en diffère par un trait singulier: il pleut du sang. Ce prodige de la nature, attesté dans l'Histoire naturelle de Pline l'Ancien et dans les Histoires de Grégoire de Tours ${ }^{24}$, ne suscite qu'un bref commentaire d'Alexandre, qui l'interprète comme un signe de la colère des dieux qui présage leur perte, mais qui, malgré son étrangeté, n'arrête ni le voyage des Grecs ni le mouvement strophique. Le narratif l'emporte sur le lyrique. Ce traitement du thème de l'orage peut s'expliquer par son utilisation comme une forme jointe et non éclatée comme dans les exemples antérieurs. La description de la tempête est très brève et suivie. Elle point sur une montagne; quatre nuages vermeils provoquent un grand fracas et des gouttes de sang tombent sur les troupes (vv. 35723577). La tempête s'intègre dans l'exploration d'Alexandre comme un nouveau danger qui, loin de le détourner de son objectif de rencontrer la fontaine de jeunesse, renforce ses propos:

Alixandres en jure les dieus ou il s'atent

Ne laira por mal pas ne por perillement

Ne voie la fontaine se Dieus le li consent. (vv. 3595-3597)

(24) «Praeter haec inferiore caelo relatum in monumenta est lacte et sanguine pluisse M. Acilio C. Porcio cos. et saepe alias, sicut carne P. Volumnio Servio Sulpicio cos., exque ea non perputruisse quod non diripuissent aves, item ferro in Lucanis anno ante quam $\mathrm{M}$. Crassus a Parthis interemptus est omnesque cum eo Lucani milites, quorum magnus numerus in exercitu erat», Pline l'Ancien, Histoire naturelle, C. MaYhoff, éd., C. Plini Secundi. Naturalis Historiae, Livri XXXVII, Lipsiae, In aedibus B. G. Tevbneri, 1967, L. II-56. Grégoire de Tours, Histoires, Livre VI, 14: «De portentis quae apparuerunt. [...] mense Ianuario pluviae, coruscationes atque tonitrua gravia fuerunt; flores in arboribus ostensi sunt. Stilla, quem comitem superius nominavi, apparuit, ita ut in circuitu eius magna nigrido esset; et illa, tamquam se in foramen aliquod posita, ita inter tenebras relucebat, scintillans spargensque comas. Prodebat autem ex ea radius mirae magnitudinis, qui tamquam fumus magnus incendii apparebat a longe. Visa est autem a partem Occidentis in ora noctis prima. In die autem sanctum paschae apud Sessionas civitatem caelum ardere visum est, ita ut duo apparerent incendia; et unum erat maior, aliud vero minor. Post duarum vero horarum spatio coniuncta sunt simul, factamque pharum magnam, evanuerunt. In Parisiaco vero terminum verus sanguis ex nube defluxit et super vestimenta multorum hominum caecidit et ita tabe maculavit, ut ipsi propria indumenta horrentes abnuerunt. Tribus enim locis in termino civi- tatis illius hoc prodigium apparuit. In Silvanectinse vero terreturio hominis cuiusdam domus, cum ille mane surgerit, sanguine respersa ab intus apparuit»; MOnumenta Informatik, 'monumenta.ch', http://monumenta.ch/ latein/?domain=\&apparat $=\&$ lang $=0$, accès: 30.04 2012; [... on eut, dans le mois de janvier, des pluies, des éclairs et de violents tonnerres; on vit des fleurs sur les arbres. Il apparut dans le ciel une étoile à laquelle j'ai donné plus haut le nom de comète. Le ciel, tout autour, était profondément obscur, en sorte que, placée comme dans un creux, elle reluisait au milieu des ténèbres, scintillait, et étalait sa chevelure: il en partait un rayon d'une grandeur merveilleuse, qui paraissait au loin comme la fumée d'un grand incendie; on la vit à l'occident, à la première heure de la nuit. On vit aussi dans la ville de Soissons, le saint jour de Pâques, le ciel ardent, comme s'il eût été embrasé de deux incendies: il y en avait un plus grand, et l'autre moindre. Au bout de deux heures, ils se réunirent, et, après avoir formé comme une grande flamme, ils disparurent. Dans le territoire de Paris, il tomba des nuages une pluie de sang véritable: beaucoup de gens la reçurent en leurs vêtements, et elle les souilla de telles taches qu'ils s'en dépouillèrent avec horreur. Le même prodige se manifesta en trois endroits du territoire de cette cité. Dans celui de Senlis, un homme, en se levant le matin, trouva l'intérieur de sa maison arrosé de sang] Grégoire interprète la pluie de sang comme un présage funeste qui annonce des épidémies. 


\section{Conclusion}

Dans les deux romans d'Alexandre le motif de la tempête s'inscrit dans l'épisode indien et se place après la traversée des colonnes d'Hercule, c'est-à-dire dans le temps de la transgression par Alexandre de l'interdit des dieux. Par conséquent, les épisodes et les espaces qui s'ensuivent sont marqués par cette empreinte maléfique. La séquence d'événements est plus serrée dans Le Roman d'Alexandre d'Alexandre de Paris par rapport à celle de Thomas de Kent, plus encyclopédique. Les trois versions de ce thème alternent avec d'autres aventures et des rencontres extraordinaires selon l'ordre suivant sur la «scène» de la vallée périlleuse:

1. «le tempier», filles de l'eau, fontaines de résurrection et d'immortalité;

2. «l'estorbellons», rencontre des ichtyophages, filles-fleur;

3. «le torment» de sang, la fontaine de jeunesse, les arbres du Soleil et de la Lune.

C'est la partie du roman où les mirabilia de l'Orient l'emportent sur les motifs épiques. L'espace, fragmenté en petites cellules individualisées mais identiques dans leur sens profond, demeure toujours hostile au héros macédonien, comme la météorologie et les femmes. Dans cet itinéraire, il perd l'espoir de s'en approprier de même que la fontaine de l'immortalité se dérobe à ses yeux et que les arbres du Soleil et de la Lune mettent un terme bref à sa vie. La domination de l'espace et du temps échappe à Alexandre.

Chaque auteur révèle un traitement différent de la tempête conforme au caractère spécifique de son roman. Thomas de Kent façonne la description de la tempête comme une digressio aux contours bien définis qui constitue une pause dans le récit, comme les descriptions des peuples de l'Inde, d'Éthiopie ou de l'île de Taprobane (Ceylan). Alexandre de Paris, par contre, intègre la tempête dans le récit et la transforme en une aventure qui met à l'épreuve le courage et l'endurance des Grecs et notamment de leur souverain. Mais tous les deux développent un jeu d'amplification de la tempête par rapport aux modèles latins, que ce soit de la poésie «maritime» ou du fragment de l'Epistola Alexandri ad Aristotelem. La description déborde la frontière de la laisse pour se poursuivre à travers plusieurs strophes consécutives qui scandent les clichés des météores, du comportement des hommes et de l'embellie. Seule la description du «torment» de la laisse 201 d'Alexandre de Paris se complète en une unique laisse à cause de sa brièveté. La forme disjointe ou éclatée l'emporte sur la forme jointe.

La désagrégation de la tempête assouplit sa fusion avec des motifs, soit narratifs - rencontre avec les géants fendus jusqu'au nombril, grotte enchantée, guides des fontaines et des arbres - qui accélèrent le rythme du récit, soit lyriques comme le planctus, qui le ralentissent. Il en résulte un portrait complexe et contradictoire du héros macédonien qui hésite entre la déroute et l'impuissance face à la météorologie adverse, et l'orgueil et le défi aux dieux. Les tempêtes survenues dans le val périlleux dépouillent Alexandre de sa fonction royale et découvrent sa nature humaine et mortelle. Thomas de Kent et Alexandre de Paris réécrivent la description de la tempête à partir d'un exercice d'amplification et d'allongement qui fait appel aux motifs du planctus, à l'esthétique de la répétition épique et aux mirabilia de l'Orient romanesque. 
Annexe I

Thomas de Kent, Le Roman d'Alexandre ou Le Roman de Toute Chevalerie

359

5780 Cerché ont les regnés tresques al chef aquilon

E tresqu'en orient de la grant mer de Claustron.

Ore vont vers occident cerchant la region.

Sur une grant rivere sunt logé li baron;

En un mont sunt tendu tref e pavillon.

5785 Assis sunt a manger, car bien orent saison,

Quant sodeinement lur surd une tempesteison,

Unes neires nues devers septentrion;

Avougly les regnés sur eaux [e] environ

Qe nul ne pot veer fors flambe de charbon.

CXLIIII De la tempeste qe survint a Alisandre.

360

5790 Devers l'occident lur surd tempeste sodeinement

De toneire e d'escleire e d'un orible vent

Qe descire les trefs, les tentes ensement.

Depescent ces cordes, rompent cil garnement.

Le feu vole de l'eir qe les bestes esprent.

5795 N'ad si hardy qe donc ne se espoent.

Donc dient li baron, le pople e la gent:

'C'est a mult bon dreit qe nous aiom turment!

Nous errom contre Dieu e contre son talent.

Ses secrez encerchom, fesom malement;

5800 E ceo volom enquerre par nostre hardement

Qe Dieux a mortel home ne granta nient.'

Quant roys Alisandre ceste parole entent:

'Seignurs barons', dit il, 'n'aiez marrement!

Decembre est en yver, ceo sachez veraiment.

5805 L'orage est de saison, le temps le consent.

N'est pas de l'ire Dieu, ne soiez pas dolent,

Ainz eimes en un mont herbergé malement.

Ostez de cy les trefs tost e ignelement;

En cest val les fichez, si serrom surement.'

5810 Sergant e mareschal font son comandement.

Chescun oste son tref e jus al val le tent.

A peine les ostent tant ventolt asprement.

Herbergé sunt li ost al val seurement,

Asseent al manger, pernent le liement.

5815 En poy d'oure muee sunt li quatre element,

$E$ fet un froit trenchant e nege forment,

Une neif si liee cum toison de bident.

Mult ert grant merveille si ele nes acravent.

361

La noif fu hiduse, le freit grant qui les destreint.

5820 Mult par est malbailliz qui a descovert l'atent.

Grisil chiet ovec qe les testes lur fent;

Li tref, li pavillon sunt covert e teint.

Tot ly feu de l'ost sunt por poy esteint.

Enpirent li haubert e li escu a or teint.

5825 Donc prient Dampnedieu qe autre orré lur present; 
Escrient e plorent, a genoilz prient meint.

Est lur donques un orages qe toz les autre[s] veint!

Une nue de ciel qe feu e flambe enpeint.

Escrie le pople: 'Ceo font fere ly seint!'

5830 Ore ad Alisandre pour; oianz toz s'en pleint.

362

Hiduse e laide e horible fu la nue;

Unques puis icel temps ne fu itel veue.

Chiet en feu e flambe, e fudre en est chaue;

Broille les pavillons e les bestes lur tue.

$5835 \mathrm{Li}$ arbre en sunt chaeit, l'erbe est abatue,

La terre environ est ardante aparue.

Grant peine aprés autre lur est ore acreue.

Meinte defense i ot pur la fudre tendue.

Le chef Alisandre coverent d'une targe a or batue.

5840 Alisandre escrie: 'Dieu du ciel, nostre aiue!

Moult par est icil fols qui tiel Roy irascue.

Bien deit home aver pour quant sa ire est conue.'

Mult se pleint Alisandre, de vie en ad dotance.

Ses homes veit morir, pur ceo est en balance.

5845 Il tint l'escu drescé, mes ne quert nul[e] lance.

'Sire,' dit Tholomeu, 'n'aiez en quer pesance.

Ces perils, ces tormenz pernez toz en suffrance.

Cil est Dieux sovereins qui tieux darz nos lance.

Ore poez bien veer qe poy vaut surquidance,

5850 Orgoil ou chevalerie contre la Dieu puissance,

Car mortele est chescune terriene substance.

Depriom Dampnedieu e sa chere semblance

Qu'il ait merci de nous e doint nos sa creance,

E des tormenz qe nous avom nous face demorance.'

\section{4}

5855 Del feu e de la fudre cil de l'ost pour ourent, E requistrent Dieu, toz en oreisons jurent.

Donc cesse la tempeste, e la gent se asseurent.

Par le comand Alisandre mangerent puis e burent.

Treis jurs i sojornerent qe unques ne se murent,

5860 Car les noires nues desur lur chefs esturent.

Cink cenz chevalers del torment occis furent;

Ly vifs les enterrerent al plus bel qu'il surent.

Puis vont vers occident tant cum li desert durent;

Cil les guient devant qe les terres conurent. 
Annexe II

Le Roman d'Alexandre d'Alexandre de Paris

156

Qant li rois fu remés et li os s'en repaire,

Maintenant aprés ce, que il ne tarda gaire,

Si commence a touner et foudroie et esclaire,

2708 Li mons prist a crauller, li vaus desous a braire,

Et gete une puor dont li rois sent la flaire.

A ciaus qui s'en tomerent n'avint il nul contraire

Fors de lor bon segnor qui remest a mal traire;

2112 Por celui font tel duel ja mais ne sera maire,

Cel jor i ot rompu mainte pelice vaire.

Desus un mabre bis, dont estoit plaine l'aire,

Sist li rois qui tous dis fu de biens essamplaire.

2716 La paor quit destraint li change le viaire,

Car qui de mort se crient bien est drois qu'il i paire,

Tous en est enpalis, car il ne set que faire.

\section{7}

Qant li rois vit la terre en pluisors lieus ardoir,

2120 Les montaignes crauller et les roches movoir,

Et sovent esclistrer et les pierres chaoir,

Se li rois ot paour ne fait a mentevoir.

Ses compaignons regrete par mervelleus savoir,

2724 Emenidon sor tous, en cui ot son espoir.

«Ha! gentieus chevaliers, de nobile pooir,

Sages, preus et cortois, humeles et dous por voir,

Car onques a nul jor ne me poi percevoir

2728 Que orgeus vos creüst por richece d'avoir Ne soufraite meïst vostre oevre en nonchaloir. Je ne poi par mon cors plus d'un home valoir, Mais vostres grans barnages se fist tous jors paroir

2732 Et moi segnor de terre et largement tenoir, Et je refis molt bien tout a vostre voloir. Ce que je part de vos me fait le cuer doloir. Ahi ! franc compaignon, dolent par estovoir,

2736 Com vos est grans dolors venue des hersoir. Hier fustes vos en joie, hui est de blanc en noir. Sans segnor repairiés, ci m'estuet remanoir Dolans et esgarés, pres de mort recevoir.»

\section{8}

2740 Qant li rois fu remés, tous seürs de morir, Maintenant commença li jors a oscurcir, Li solaus a changier et li tans a noircir, Forment a esclistrer et li cieus a partir.

2744 Li mons prist a crauller et li vaus a fremir, Et gete unes teniebres o flairor de püir. Bucifal ne se pot en estant soustenir, Ne li rois en estant, ains le convint gesir.

2748 Ainc Dieus ne fist mervelle dont li puist sovenir, Fiere, laide et hideuse, que on doie cremir, Dont ne voie entor soi grans batailles tenir: Les dragons fu getans qui font l'erbe bruïr

2752 Et grans serpans volans qui font l'air escroissir 
Et maufés rechingnans quil veulent assaillir

Et font as cros de fer samblant de lui saisir.

Lors n'ose Bucifal ne grater ne henir,

2756 Sous le mantel le roi met son chief por covrir,

Q'il nes ose veoir ne ne queroit sentir,

Et ne por qant li rois s'apense du ferir.

Onques de cel deduit ne li vaurent faillir

2760 Entresques au matin que il vit esclarcir.

En tant d'eure c'on puet un oel clorre et ovrir

Ne sot il que devinrent ne n'en pot un choisir;

Ne por qant molt fu liés qant le vaurent gerpir,

2764 Car de lor compaignie ne pooit il joïr.

Molt par sera grans joie s'encor en puet partir,

Encor feroit li rois barnage resbaudir.

159

Ses gentieus compaignons Alixandres recoie

2768 Et dist que de bien faire les avoit mis en voie. «Se fortune ne fust qui les choses desvoie, La terre, icel petit que Dieus en fist, fust moie, Car puis q'Emenidon a mon costé avoie

2772 Ou devant ou derrier pres de moi le savoie, Force de nule gent el siecle ne cremoie; Se ici ou aillors de lui parler ooie,

Bien sai que grant dolor ne mal ne sentiroie;

2776 S'il el bien se maintient que veoir i soloie, Tous iert sires du mont et mes cuers li otroie. Las! ne l'en puis aidier, car cis vaus me guerroie Et li dieu qui m'ont pris, dont je ne me gardoie.

2780 Se g'iere en ingal cort et mes armes avoie, De laide traïson trestous les proveroie,

De l'ire que j'en ai sor aus me vengeroie».

160

Alixandre est el val, ne se set conseillier,

2784 Il ot la terre bruire, si craullent li rochier; Bien set de l'eschaper ne li est pas legier, Ses gentieus compaignons prist molt a recoier. Li Grieu, qui sor la mer s'estoient fait logier,

2788 Onques la nuit ne vaurent ne boire ne mangier, Ains regretent et pleurent lor segnor droiturier, Le bon roi Alixandre, qui tant fist a proisier, Et deprïent les dieus et vont sacrefier

2792 Q'Alixandre garissent de mort et d'encombrier. Et li rois fu el val, n'i ot que corecier, Il ne set en quel guise se doive conseillier, Comment de la prison se puisse deslier.

2796 Il regarda a mont si vit l'air espessier Et les nues torbler et le tans a changier, $\mathrm{Ne}$ fu mie a seür qant il vit tel tempier. S'il ot peor ne doute, ne fait a mervellier,

2800 Bonement commença les dieus a deproier Qu'il li soient garant, car il en a mestier. Du pan de son hermine covert d'un paile chier Envolepe sa teste et tint son branc d'acier

2804 Et tint bien par la resne Bucifal son destrier. 
Au matin commença li solaus a raier;

Lors saut en Bucifal Alixandres d'Alier

Et commença li rois tout le val a cerchier.

2808 Joste une grant montaigne commence a chevauchier

Et vit une cisteme qui resamble celier.

El n'estoit mie ouvree de chaos ne de mortier,

Mais li dieu l'orent faite en la roche entaillier.

2812 En mi ot un perron qui plus iert durs d'acier,

Lai ens sous cele pierre avoit un aversier

Felon, qui cuidoit faire tout le mont perillier.

$\mathrm{Li}$ rois i est entrés, la croute veut cerchier,

2816 Qu'il veut toutes les choses prover et ensaier.

N'i vit home ne feme ou se puisse acointier.

Icele male chose le prist a araisnier,

Sous la pierre ou estoit commença a huchier:

2820 «Sire, rois Alixandres, car me venés aidier,

Et je t'ensegnerai or endroit le sentier,

Comment porras issir de cest val de legier,

Si que ja n'i perdras la monte d'un denier».

2824 Li rois entent la vois, prist soi a mervellier,

Paor ot de la mort, molt se crient d'engignier.

$$
* * *
$$

181

Molt fist bien Alixandres qant ciaus d'Ynde a creüs,

Toute la matinee s'en est li rois meüs

3132 Por retomer arriere la dont il iert issus,

En la fastïene Ynde dont il estoit venus.

Ja estoit li fus grans, et li rois descendus

A un estanc qu'il truevent entre tertres agus

3136 Qui tous estoit plains d'eaue, mais sempres fu beüs

Des chevaus et des bestes que avoit recreüs

Li chaus qui du soleil estoit si grans cheüs.

D'autre part de la rive ot homes parcreüs,

3140 Chascuns iert par le cors dusq'au nombril fendus

Et environ le dos comme bestes velus.

Des Grieus se mervellierent qant il les ont veüs;

Molt par les veïssiés dolens et irascus

3144 Qant veü ont teus homes, qu'il n'ont pas conneüs;

Vers aus vinrent corant, molt fu grans lor vertus.

Ainc ne vestirent drap qui fust fais ne tissus,

Tuit ensamble lor lancent pierres et peus agus,

3148 Plus de cinc cens sergans lor i ont mors rendus.

Cil de l'ost s'estormissent ses eüssent seüs,

Qant uns estorbellons lor est devant venus

Qui tres et pavellons lor i a abatus,

31S2 Les somiers trebuchiés et de tisons ferus;

Peliçon et mantiaus i ont assés perdus,

Entresi vers le ciel en porte lor escus.

Aprés icest domage lor est autres creüs:

$31 \mathrm{~S} 6$ Ensement comme noif est fus du ciel pleüs,

Que toute arst la contree ensement comme fus.

Or dient tuit par l'ost: «Libers est irascus,

Ou il ou Erculés font ore ces vertus,

3160 Par la consence as dieus nos est cis maus venus». 
182

Qant li vens fu cheüs et li estorbellons,

A mont envers le ciel en guise de tisons

Virent venir les rais ardans comme brandons.

3164 Cil de l'ost s'estormissent, qui criement les charbons,

De lor escus se cuevrent, ce fu lor garisons;

Et cil qui nul n'en orent se tinrent por bricons,

Brullees ont les testes et barbes et grenons.

3168 Qant du ciel fu cheüe la rougor et l'arsons,

A negier commença de l'air qui fu enbrons;

$\mathrm{Ne}$ demora puis gaires qu'en vint si grans foisons

Que li flocel chaoient si grant comme toisons,

3112 La noif iert sor les tres haute comme dongons.

Alixandres commande a trestous ses barons

Que ne remaigne en l'ost escuiers ne garçons

Qu'il ne mainent les bestes par tous les pavellons

3176 Et abatent la noif a peus et a bastons.

Por la chalor des bestes fu grans remetoisons,

La noif qui iert remise chaucha comme sablons.

Aprés lor vint de pluie si grant sorjonsions

3180 Que tout en porte a val la noif et les glaçons.

Li estans rempli d'eaue, qui iert et grans et lons.

\section{3}

Quatre jors fu li rois que d'iluec ne se mut

Et fist molt grant froidure, assés nega et plut;

3184 Ainc l'espoisse de l'air por ice ne descrut Alixandres demande a ciaus d'Ynde que dut Q'aprés si grant chalor si grant froidure mut, Mais onques por le dire nus d'aus ne s'aparut.

\section{4}

3188 Deus viellars yndiens ont es desers trovés Qui ont longes les barbes jusqu'au neu des baudrés.

Li rois lor a enquis: «Qui estes, ou alés?»

Cil li ont respondu: «Ci est nostre regnés,

3192 Des desers de Rimost est nostre naïtés.

Li qels est Alixandres? Et car le nos mostrés,

Car veoir le volons plus qu'ome qui soit nés,

Por ce que sor tous homes est cremus et doutés».

3196 Li chevalier lor dient: «Toute l'ost esgardés,

A celui qui mieus samble segnor si vos tenés».

Cil esgardent le roi, qui fu gros et quarrés,

Large ot 1'enforcheüre si fu bien figurés;

3200 Autresi le connurent com s'il lor fust mostrés. «Sire, font li viellart, se en nos vos fiés,

As arbres vos menrons que vos tant desirrés,

Qui vos diront noveles de la mort q'atendés».

3204 Qant Alixandres l'ot, ses en a merciés,

Riches conrois lor done et bons muls sejornés.

185

«Segnor, dist Alixandres, je vos commant et reu:

Remuons nos herberges et querés autre leu,

3208 Car je voi molt le ciel et pale et ynde et bleu.

Il ne nos promet mie ne seürté ne geu, 
Ains sont irié li dieu qui nos ont mis en freu,

Tel chose m'ont hui faite dont ai au cuer grant neu,

3212 Car set vins chevaliers, qui molt estoient preu,

I avons hui perdu de la noif et du feu.

Sevelissons les mors et si lor faisons veu;

Por le maingier haster voisent avant li qeu».

186

3216 Les mors ont sevelis, li Grieu s'en sont torné.

Li quieu s'en vont avant, le mengier ont hasté;

D'un grant tertre ou il ierent ont un val avalé

Et truevent as herberges le mangier apresté;

3220 A pié s'en vont deduire quant il orent disné.

Desous une grant roche truevent un lieu cavé

Ou Artus et Libers avoient conversé;

Et orent icel lieu beneoit et sacré,

3224 Que puis n'i entra hom qu'il s'en furent torné

Q'on n'eüst au tierc jor ça dehors mort trové;

Alixandres mescroit qu'il n'i ait enferté

De serpent ou d'oisel qui l'ait envenimé.

3228 Ains nel creï li rois des qu'il l'ot esprové,

Entrer i fist quatre homes qui bien furent armé,

Au tierc jor furent mort tout quatre hors trové,

En travers l'un sor l'autre a la terre geté.

3232 Alixandres les voit, longement a pensé;

Por ce qu'il n'i pert caus ne il ne sont navré,

N'en pot onques savoir li rois la verité

Comment il ierent mort et iluec aporté.

3236 Les viellars yndiens, qui du païs sont né

Et avoient grant piece el païs conversé,

A fait li rois venir si lor a demandé,

Lun des deus avant l'autre a forment conjuré,

3240 Que de cele mervelle qui l'a si effreé

Li dient ambedui toute la verité:

S'en la cave a fantosme, serpent ne enferté

Qui si soutainement a cest lieu deserté.

3244 Li dui viellart respondent, qui sont de grant aé, Que Artus et Libers par lor grant deïté

Entoschierent le lieu si l'ont enfantosmé.

Li rois ot les viellars qui ce li ont conté,

3248 Por le païs qu'il sevent a molt grant volenté

Li rois d'aus retenir, car par tout ont alé;

Et dist que des or mais seront molt si privé

Et molt seront par lui servi et honoré.

$3252 \mathrm{Li}$ viellart l'en mercient si li ont afié

Que par tout le menront a molt grant salveté.

\section{7}

Qant li vens fu cheüs et la noif fu fondue

Et li estans fu plains de l'iaue qu'iert cheüe

3256 Et de la grant mervelle qui du ciel fu pleüe, Qui par icel convers fu ainsi espandue Et de trestoutes pars en l'estanc acourue Deus piés plus haut la rive de cele qu'iert beüe,

3260 Ja qui la lor contast par aus ne fust creüe Se il apertement ne l'eüssent veüe. 
Li jors fu biaus et clers et fist ombre sous nue.

Alixandres monta, toute l'ost se remue

3264 Et monterent un tertre dont la roche est agüe. Onques mais n'i ot sente ne chariere batue,

Por l'angoisse du chaut tous li plus fors tressue.

Li viellart vont avant qui la terre ont seüe,

3268 Et ont el cuing du val une forest veüe

Qui bien estoit dedens et garnie et vestue

De toute icele riens qui a cors d'ome aive.

$$
* * *
$$

201

Li rois issi du bos et si home ensement,

Les puceles les gïent tant com li ombres tent.

Qant ne porent avant, si souspirent forment,

3548 A terre s'agenollent voiant toute la gent

Et clinent Alixandre du chief parfondement,

A Dieu le commanderent qui le maint sauvement.

Li dui viellart les guïent par un adrecement

3552 Entre quatre montaignes par un val freolent;

Chascun an par nature i naissent cinc serpent

Qui getent fu et flambe, la terre art et esprent.

En icele valee se combatent li vent,

3556 En tous tans i a froit et gele si forment

Onques solaus n'i luist, se li livres ne ment.

Iluec ot malvais siecle qui ot froit vestement, Unes piaus i vausissent set mile mars d'argent,

3560 Car tout li mieus vestu trembloient dent a dent. Puis truevent les mervelles des desers d'Orïent, Couluevres et serpens qui mordent aigrement.

Qui la ot bones armes nes donast son parent,

3564 Ains les tint pres de soi et son cors en deffent. N'i vausist chascuns estre por 1'or de Bonivent, Car la terre sous aus peçoie et craulle et fent Et li cheval i entrent tresq'a l'afeutrement;

3568 Tuit li mellor destrier i devenoient lent. Iluec fist grant folie qui a pié i descent, Car la terre lor faut desous lor piés et ment; Le jor en i remerent, ce m'est vis, plus de cent.

3572 Par mi une montaigne choississent un torment, Quatre nues vermelles par devers Occident Qui menoient entr'eles un tel tornoiement, Tel noise et tel bataille et tel tanbuissement

3576 Et plevoient le sanc ensi apertement Que les goutes vermelles chaoient sor la gent. N'i ot nul qui n'eüst sanglent son vestement. Trestous li plus hardis i mua son talent.

3S80 Li rois en apela Tholomé son parent, Cliçon, le fil Caduit, et Perdicas le gent. «Segnor, fait Alixandres, par le mien ensïent Cis signes senefie molt grant destruiement.

3584 Li dieu nos volent mal, trop alons folement». $\mathrm{Li}$ dui viellart ques guient li ont dit belement Qu'il face l'ost remaindre si voist priveement Por veoir les deus arbres devins isnellement

3588 Et la chiere fontaine et l'eaue de jovent, 
La ou renjovenissent toute la vielle gent

En l'aé de trente ans, por voir sans doutement, Qui quatre fois s'i baigne et contient sagement;

3592 Mais bien se gart de plus et de mains ensement, Car qui le devié passe n'i gaaigne noient;

Molt en a grant mervelle li rois quant il l'entent.

Alixandres en jure les dieus ou il s'atent

3596 Ne laira por mal pas ne por perillement

Ne voie la fontaine se Dieus le li consent. 